Article

\title{
Chirality Construction from Preferred $\pi-\pi$ Stacks of Achiral Azobenzene Units in Polymer: Chiral Induction, Transfer and Memory
}

\author{
Tengfei Miao ${ }^{1}$, Lu Yin ${ }^{1}$, Xiaoxiao Cheng ${ }^{1}$, Yin Zhao ${ }^{1}$, Wenjie Hou ${ }^{1, *}$, Wei Zhang ${ }^{1, *}$ () and \\ Xiulin Zhu ${ }^{1,2}$ \\ 1 Suzhou Key Laboratory of Macromolecular Design and Precision Synthesis, Jiangsu Key Laboratory of \\ Advanced Functional Polymer Design and Application, State and Local Joint Engineering Laboratory for \\ Novel Functional Polymeric Materials, College of Chemistry, Chemical Engineering and Materials Science, \\ Soochow University, Suzhou 215123, China; mtf0824@163.com (T.M.); yinlu0701@163.com (L.Y.); \\ studentscheng@163.com (X.C.); zhaoyinsuda@126.com (Y.Z.); xlzhu@suda.edu.cn (X.Z.) \\ 2 Global Institute of Software Technology, No 5. Qingshan Road, Suzhou National Hi-Tech District, \\ Suzhou 215163, China \\ * Correspondence: lionhoumail@hotmail.com (W.H.); weizhang@suda.edu.cn (W.Z.); \\ Tel.: +86-512-6588-4243 (W.Z.); Fax: +86-512-6588-2787 (W.Z.)
}

Received: 16 May 2018; Accepted: 1 June 2018; Published: 4 June 2018

\begin{abstract}
The induction of supramolecular chirality from achiral polymers has been widely investigated in composite systems consisting of a chiral guest, achiral host, and solvents. To further study and understand the process of chirality transfer from a chiral solvent or chiral molecules to an achiral polymer backbone or side-chain units, an alternative is to reduce the components in the supramolecular assembled systems. Herein, achiral side-chain azobenzene (Azo)-containing polymers, poly(6-[4-(4-methoxyphenylazo) phenoxy] hexyl methacrylate) (PAzoMA), with different $M_{\mathrm{n}}$ s, were synthesized by atom transfer radical polymerization (ATRP). Preferred chirality from supramolecular assembled trans-Azo units of PAzoMAs is successfully induced solely by the neat limonene. These aggregates of the polymers in limonene solution were characterized by circular dichroism (CD), UV-vis spectra, and dynamic light scattering (DLS) under different temperatures. The temperature plays an important role in the course of chiral induction. Meanwhile, supramolecular chirality can be constructed in the solid films of the achiral side-chain Azo-containing polymers that were triggered by limonene vapors. Also, it can be erased after heated above the glass transition temperature $\left(T_{\mathrm{g}}\right)$ of the polymer, and recovered after cooling down in the limonene vapors. A chiroptical switch can be built by alternately changing the temperature. The solid films show good chiral memory behaviors. The current results will facilitate studying the mechanism of chirality transfer induced by chiral solvent and improve potential application possibilities in chiral film materials.
\end{abstract}

Keywords: supramolecular chirality; Azo-containing polymers; chiral aggregation; chiral film

\section{Introduction}

Chirality is a common phenomenon in nature and organisms, such as spiral amino acids, vines, conches, and even the galactic system [1]. The study of chirality in terms of the elemental distribution and molecular structure has always attracted the curiosity of scientists [2]. At the supramolecular level, supramolecular chirality refers to chirality generated by non-covalent interaction between building units, such as hydrogen bonding [3], $\pi-\pi$ stacking [4,5], electrostatic interaction [6], and host-guest interaction [7]. The production of supramolecular chirality from achiral polymer 
building blocks has very important significance in theoretical research and practical application. Many strategies, such as chiral liquid-crystal field [8], chiral solvent [9-11], circularly polarized light (CPL) [12-14], interfacial interaction [15], and gelation [16] have been reported to produce supramolecular chirality from achiral polymer systems.

The chiral induction of achiral substances by chiral solvent is a flexible and effective method, which is suitable for organic small molecules [17], oligomers [18], and polymer systems [19,20]. The first example of a chiral-solvation-induced chirality of an achiral polymer was demonstrated by Green et al. [21]. The dynamically interconverting helical senses can be found in the circular dichroism (CD) spectrum when dissolving achiral polyisocyanate in several chiral chlorinated solvents. Motivated by this pioneering study, optically active polymers or polymer aggregates, such as polysilanes [22], polyfluorenes [23-25], polyacetylenes [26], and azobenzene (Azo)-containing polymers [9,27] have been shown to achieve chirality in aggregation states induced by chiral solvation. Azo-containing polymers are very promising materials in the field of photoswitchable molecular systems and liquid crystalline materials. Due to the dramatic changes in the polarity, shape, and size of the Azo units during the process of photoisomerization, Azo-containing polymers showed unique behaviors of chiroptical switching, which was observed by UV-vis and circular dichroism (CD) spectra [28]. In our group, we have introduced Azo groups into the main chain [9] and side-chain [27] of the achiral polymers, and successively achieved the supramolecular chirality from the achiral building blocks induced by chiral limonene. However, this induction and assembly process of main chain Azo-containing polymers requires a complex system consisting of the good solvent, a chiral solvent, and a weak solvent. Recently, we further simplified the assembled components of a side-chain Azo-containing polymer by using limonene as the weak and chiral solvent simultaneously $[27,29,30]$. On the one hand, although much effort has been paid to simplifying the assembled components and studying the inducing mechanism, we still have a problem remaining to be solved of whether the construction of supramolecular chirality in the achiral side-chain Azo-containing polymer can be achieved by pure limonene under the controlled temperature. The neat chiral solvent will avoid the interactions between the other solvents (good and poor) and building blocks.

On the other hand, the chirality transfer and amplification phenomena have been generally observed in polymer solution [22,31,32]. However, the chiral transfer, chiral switching, and memory of supramolecular chirality in solid polymer films are more promising in practical applications, such as chiral nonlinear optics and data storage. Guerra et al. [33] presented that the chiral s-PS (syndiotactic polystyrene) films can be obtained through inducing by chiral limonene molecules and then replacing them with their achiral counterparts. The use of circularly polarized light (CPL) has been demonstrated as a method for the chiral induction [34] and chiral regulation [13] of polymer films. Wu et al. reported that the photoinduced circular dichroism of polymer liquid crystals on the thin films can be erased by heating the films above the clearing temperature or by annealing the films in the liquid-crystalline phase [35]. However, the chiral induction of side-chain Azo-containing polymer films by chiral solvent vapors and the modulation of chirality by variable temperature was rarely reported.

In this work, we present the construction of supramolecular chirality from the aggregation of an achiral side-chain Azo-containing polymer in the solution state induced by pure limonene, and in the solid films triggered by chiral limonene vapor. Through a heating-cooling treatment of the polymer solution, the chiral aggregation of achiral Azo-containing polymers can be effectively generated. In the solid film, the chiral limonene vapors can also induce well-organized stacks of Azo units, forming the supramolecular chirality. Meanwhile, the CD signal of the polymer films can be readily erased after heating above the glass transition temperature $\left(T_{\mathrm{g}}\right)$ of the polymer, and recovered after cooling it down in the chiral solvent vapors. This regular pattern of chiroptical switch can be repeated effectively at least five times. Besides, the supramolecular chirality of the polymer film can be memorized well. 


\section{Experiment}

\subsection{Materials}

1-Chloro-6-hydroxyhexane (Acros, 95\%), (R)-(+)-limonene $\left(1 R, \mathrm{TCI},>95 \%,[\alpha]_{589}^{24}=+99.62^{\circ}\right.$, TCI Shanghai Development Co., Ltd., Shanghai, China) and (S)-(-)-limonene (1S, TCI, >95\%, $[\alpha]_{589}^{24}=-97.72^{\circ}$, TCI Shanghai Development Co., Ltd., Shanghai, China) were used without further purification. Copper (I) bromide (CuBr, Aldrich, St. Louis, MO, United States, 98\%) was purified with glacial acetic acid and washed with pure ethanol, and then stored under argon before use. Ethyl 2-bromoisobutyrate (EBiB) (TCI, 98\%), $N, N, N^{\prime}, N^{\prime} N^{\prime \prime}$-pentamethyldiethylenetriamine (PMDETA) (Aldrich, St. Louis, MO, United States, 97\%), and the monomer, 6-[4-(4-methoxyphenylazo) phenoxy]hexyl methacrylate $\left(\mathrm{AzoMA}_{6}\right)$ were synthesized as described previously [36], which was confirmed by the ${ }^{1} \mathrm{H}$ and ${ }^{13} \mathrm{C} \mathrm{NMR}$ data (Figure S1). ${ }^{1} \mathrm{H} \mathrm{NMR}\left(\mathrm{CDCl}_{3}, 300 \mathrm{MHz}\right),(\delta, \mathrm{ppm}): 7.87$ $(\mathrm{m}, 4 \mathrm{H}), 6.99(\mathrm{~m}, 2 \mathrm{H}), 7.26(\mathrm{~m}, 2 \mathrm{H}), 6.10(\mathrm{~s}, 1 \mathrm{H}), 5.54(\mathrm{~s}, 1 \mathrm{H}), 4.18(\mathrm{t}, 2 \mathrm{H}), 4.03(\mathrm{t}, 2 \mathrm{H}), 3.92(\mathrm{~s}, 3 \mathrm{H}), 1.98$ $(\mathrm{s}, 3 \mathrm{H}), 1.81(\mathrm{~m}, 2 \mathrm{H}), 1.71(\mathrm{~m}, 2 \mathrm{H}), 1.50(\mathrm{~m}, 4 \mathrm{H})$. Elemental analysis, calculated values for $\mathrm{C}_{23} \mathrm{H}_{28} \mathrm{~N}_{2} \mathrm{O}_{4}$ : C, 69.68; H, 7.12; N, 7.07. Found: C, 69.32; H, 6.95; N, 7.11.

\subsection{Synthesis of Poly(6-[4-(4-methoxyphenylazo) phenoxy] Hexyl Methacrylate (PAzoMA)}

AzoMA (0.5 g, $1.26 \mathrm{mmol})$, EBiB (12.3 mg, $0.063 \mathrm{mmol})$, PMDETA (10.94 mg, $0.063 \mathrm{mmol})$, $\mathrm{CuBr}(9.05 \mathrm{mg}, 0.063 \mathrm{mmol})$, and THF $(1.5 \mathrm{~mL})$ were added to a $5-\mathrm{mL}$ ampoule. Then, the ampoule was flame-sealed after being deoxygenated with three standard freeze-pump-thaw cycles. The polymerization under argon atmosphere was carried out at $70{ }^{\circ} \mathrm{C}$ for $1.5 \mathrm{~h}$. The mixture was diluted with $1 \mathrm{~mL}$ of THF, passing a column of neutral $\mathrm{Al}_{2} \mathrm{O}_{3}$, and then precipitated into an excess of methanol $(50 \mathrm{~mL})$ twice. After collection by filtration, the polymer was dried in a vacuum oven overnight at $30{ }^{\circ} \mathrm{C}(0.348 \mathrm{~g}, 69.5 \%)$. Another polymer with different $M_{\mathrm{n}}$ was prepared by adjusting the molar ratio of the monomer and initiator with the similar procedures. The molecular weights and molecular weight distributions of the obtained two polymers are listed in Table 1.

Table 1. Molecular weight characteristics of the Azo-containing polymers.

\begin{tabular}{cccccc}
\hline Entry & Ratio $^{\mathbf{a}}$ & Conv. $^{\mathbf{b}}(\mathbf{\%})$ & $\boldsymbol{M}_{\mathbf{n}(\text { th })}{ }^{\mathbf{c}}\left(\mathbf{g ~ m o l}^{-\mathbf{1}}\right)$ & $\boldsymbol{M}_{\mathbf{n}(\mathrm{GPC})}{ }^{\mathbf{d}}\left(\mathbf{g ~ m o l}^{-\mathbf{1}}\right)$ & $\boldsymbol{M}_{\mathbf{w}} / \boldsymbol{M}_{\mathbf{n}}{ }^{\mathbf{d}}$ \\
\hline PAzoMA $_{1}$ & $20: 1: 1: 1$ & 69.5 & 5500 & 7400 & 1.20 \\
PAzoMA $_{2}$ & $70: 1: 1: 1$ & 53.4 & 14,800 & 12,400 & 1.14 \\
\hline
\end{tabular}

a Polymerization ratio: $[\mathrm{AzoMA}]_{0} /[\mathrm{EBiB}]_{0} /[\mathrm{CuBr}]_{0} /[\text { pentamethyldiethylenetriamine (PMDETA) }]_{0} \cdot{ }^{\mathrm{b}}$ Determined gravimetrically. ${ }^{\mathrm{c}}$ Calculated by $M_{\mathrm{n}(\mathrm{th})}=\left([\text { Monomer }]_{0} /[\mathrm{EBiB}]_{0}\right) \times M_{\mathrm{w}, \text { monomer }} \times$ Conversion $\% .{ }^{\mathrm{d}}$ Determined by gel permeation chromatograph (GPC) according to polymethyl methacrylate(PMMA) standards in THF.

\subsection{Preparation of the Optically Active Polymer Aggregates in Solution}

A small amount of polymer solid $(0.1 \mathrm{mg})$ was added to $3 \mathrm{~mL}$ of $(R)-(+)$-limonene $(1 R)$ in a quartz cell. The suspension was stirred under $100{ }^{\circ} \mathrm{C}$ for $1 \mathrm{~h}$ to make sure the polymer was dissolved completely. The concentration of polymer repeating units is $8.42 \times 10^{-5} \mathrm{~mol} \mathrm{~L}^{-1}$. The optically active polymer aggregates were prepared after the solution was cooled down to room temperature. The other polymer aggregates were prepared in a similar way. This yellowish turbid solution of PAzoMA aggregates was employed for CD/UV-vis measurements.

\subsection{Preparation of the Polymer Solid Films}

The $10 \mathrm{mg} / \mathrm{mL}$ polymer solution was obtained by solving $10 \mathrm{mg}$ of polymer solid in $1 \mathrm{~mL}$ of $\mathrm{CHCl}_{3}$. A thin film sample of PAzoMA was prepared by spin coating about $0.1 \mathrm{~mL}$ of the polymer solution onto a clean quartz plate at a speed of $0.5 \mathrm{rpm}$ for $6 \mathrm{~s}$ and a speed of $1.9 \mathrm{rpm}$ for $20 \mathrm{~s}$. After being dried and annealed under vacuum at $90{ }^{\circ} \mathrm{C}$ for $12 \mathrm{~h}$ to drive off residual solvent, the film was stored in darkness for further study. 


\subsection{Chiral Induction Process of the Films}

The films obtained by the above method were measured with UV-vis and CD spectra. First, the film was fixed in the cell, and suspended above the surface of limonene $(0.1 \mathrm{~mL})$. Then, the vapors of limonene would be produced with the temperature increasing, and the optically active polymer film was achieved at the same time. The results of chiral assembly were recorded by UV-vis and CD spectra when the temperature was alternately changed between $70{ }^{\circ} \mathrm{C}$ and $60{ }^{\circ} \mathrm{C}$.

\subsection{Characterization}

Gel permeation chromatograph (GPC) measurements were conducted on the TOSOH HLC-8320 gel permeation chromatogragh (GPC) (Tokyo, Japan), which was equipped with a refractive index and UV detectors using two TSKgel SuperMultiporeHZ-N $(4.6 \times 150 \mathrm{~mm}, 3.0 \mu \mathrm{m}$ beads size $)$ columns (Tokyo, Japan) arranged in a series. It can separate polymers in the molecular weight range of 500-190k Da. THF was used as the eluent with a flow rate of $0.35 \mathrm{~mL} / \mathrm{min}$ at $40{ }^{\circ} \mathrm{C}$. The values of the average molecular weight $\left(M_{n}\right)$ and molecular weight distribution $\left(M_{\mathrm{w}} / M_{\mathrm{n}}\right)$ of the samples were calculated with polymethyl methacrylate (PMMA) standards. ${ }^{1} \mathrm{H}$ NMR spectra of the polymers were recorded on a Bruker nuclear magnetic resonance instrument (300 MHz, Brucker, Kalsruhe, Germany) using $\mathrm{CDCl}_{3}$ as the solvent and tetramethylsilane (TMS) as the internal standard at $25^{\circ} \mathrm{C}$. The UV-vis spectra were recorded on a UV-2600 spectrophotometer (Shimadzu (Nakagyo-ku, Kyoto, Japan)). The CD spectra were recorded on a JASCO J-815 spectropolarimeter equipped with a Peltier-controlled housing unit using a SQ-grade cuvette, a single accumulation, a path length of $10 \mathrm{~mm}$, a bandwidth of $2 \mathrm{~nm}$, a scanning rate of $200 \mathrm{~nm} \mathrm{~min}^{-1}$, and a response time of $1 \mathrm{~s}$. The samples were measured at different temperatures. The magnitude of the circular polarization at the ground state was defined as $g_{\mathrm{CD}}=2 \times\left(\varepsilon_{\mathrm{L}}-\varepsilon_{\mathrm{R}}\right) /\left(\varepsilon_{\mathrm{L}}+\varepsilon_{\mathrm{R}}\right)$, where $\varepsilon_{\mathrm{L}}$ and $\varepsilon_{\mathrm{R}}$ denoted the extinction coefficients for left and right circularly polarized light, respectively. Experimentally, the $g_{\mathrm{CD}}$ value was defined as $\Delta \varepsilon / \varepsilon=$ [ellipticity/32,980]/absorbance at the CD extremum. Elemental analyses $(\mathrm{C}, \mathrm{H}$, and $\mathrm{N})$ were measured with an EA1110 CHNO-S instrument. The thermal behavior and glass transition temperatures of PAzoMA were measured using a TA-Q100 DSC instrument (New Castle, DE, USA). Dynamic light scattering (DLS) measurements were performed with a Zetasizer Nano ZS instrument (Brookhaven, Holtsville, TX, USA) at different temperatures.

\section{Results and Discussion}

\subsection{Synthesis and Characterization of Side-Chain Azo-Containing Polymers}

The homopolymers of AzoMA (PAzoMA) (Scheme 1) were prepared by atom transfer radical polymerization (ATRP) [36] with a controlled molecular weight $\left(M_{n}\right)$ and relatively low molecular weight distribution $\left(M_{\mathrm{w}} / M_{\mathrm{n}}\right)$ (Figure S2). As presented in Table 1, the side-chain Azo-containing polymers with different $M_{\mathrm{n}} \mathrm{s}\left(7400 \mathrm{~g} \mathrm{~mol}^{-1}\right.$ and $\left.12,400 \mathrm{~g} \mathrm{~mol}^{-1}\right)$ and relative low molecular weight distribution $\left(M_{\mathrm{w}} / M_{\mathrm{n}}\right)(1.14$ and 1.20) were successfully prepared by changing the molar ratio of the monomer and initiator during the polymerization process. 


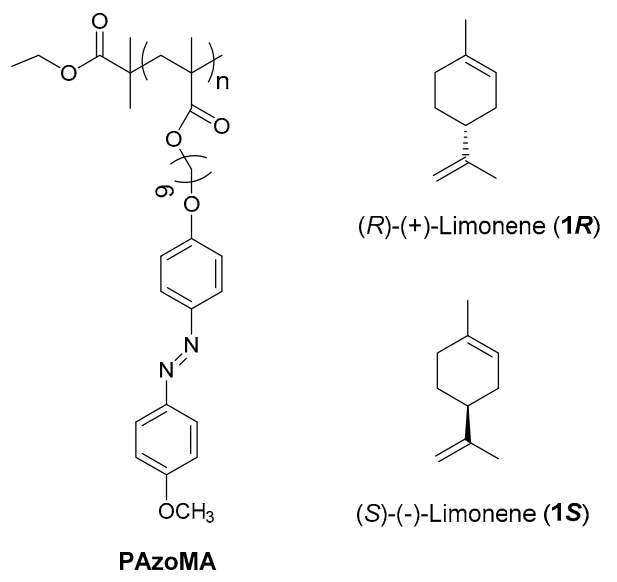

Scheme 1. Illustration of the chemical structures of poly(6-[4-(4-methoxyphenylazo) phenoxy] hexyl methacrylates) (PAzoMAs), (R)-(+)-limonene (1R) and (S)-(-)-limonene (1S).

\subsection{The Chiral Aggregation of the Azo-Containing Polymer in Neat Limonene Solution}

As was previously reported, typically a good solvent, chiral solvent, and poor solvent are required to produce supramolecular chiral aggregation from achiral conjugated polymers [27,29]. Indeed, the chiral-solvation-induced chirality by single solvent can greatly simplify the research system. In this work, the limonene ( $1 R$ and $1 S$ in Scheme 1) was simultaneously chosen as the chiral solvent, poor solvent, and good solvent. The polymer solid can be dissolved in limonene completely after stirring for $1 \mathrm{~h}$ at relatively high temperature $\left(100^{\circ} \mathrm{C}\right)$. Then, the supramolecular aggregation of the polymer occurred after cooling down the solution. The temperature is a very important factor in the supramolecular assembly of Azo-containing polymers. When the temperature is higher than $70{ }^{\circ} \mathrm{C}$, the well-dissolved polymer chains in limonene may result in a relatively weak $\pi$ - $\pi$ stacking of Azo units in polymer side chains. The optically active polymer aggregates would be produced due to a stronger $\pi-\pi$ stacking of Azo units after the temperature drops below $70{ }^{\circ} \mathrm{C}$. This result can be revealed by DLS, UV-vis, and CD spectra.

For the side-chain Azo-containing polymers $\left(\mathrm{PAzoMA}_{1}\right.$ and $\mathrm{PAzoMA}_{2}$ ), the UV-vis spectra (Figure $1 \mathrm{~b}$,d and Figure $\mathrm{S} 3$ ) of the polymer aggregates in limonene (1R or $1 S)$ consists of two absorption bands. The absorption bands ranging from $320 \mathrm{~nm}$ to $400 \mathrm{~nm}$ are attributed to the $\pi-\pi^{*}$ electronic transition of the trans-Azo, and the others from $400 \mathrm{~nm}$ to $500 \mathrm{~nm}$ are attributed to the $\mathrm{n}-\pi^{*}$ electronic transition of the cis-Azo. With the temperature decreasing, absorption of the $\pi-\pi^{*}$ band became lower and wider, indicating that the strong $\pi-\pi$ stacking of Azo units in the polymer side chain occurred.

The intense and mirror-image cotton effects can be found in the CD spectrum. The CD signals are related to the $\pi-\pi^{*}$ electronic transition of the trans-Azo, demonstrating that the supramolecular chirality could be successfully introduced to the side-chain of the Azo-containing polymers by the neat chiral solvent (limonene). As shown in Figure 1a,c,e,f, with the temperature decreasing from $70{ }^{\circ} \mathrm{C}$ to $20^{\circ} \mathrm{C}$, the $\mathrm{CD}$ and $g_{\mathrm{CD}}$ values of the aggregates began to gradually increase. The relatively poor solubility of the polymers in limonene increased the degree of aggregation, which resulted in the increasing intensity of the CD signals. These results confirm our conjecture. Compared with the chiral assembly in the mixed solvents [27], the maximum CD and $g_{\mathrm{CD}}$ values of the aggregates in neat limonene are much higher. Furthermore, $\mathrm{PAzoMA}_{2}$ with a high molecular weight gives higher $\mathrm{CD}$ and $g_{\mathrm{CD}}$ absolute maximum values (Figure 1e,f), resulting from the much higher degree of chiral aggregation. The stronger chiral aggregation behavior of $\mathrm{PAzoMA}_{2}$ compared with $\mathrm{PAzoMA}_{1}$ can be observed in their UV-vis spectra (Figure $1 b, d$ ). The relatively lower UV-vis absorption intensity and wide spectra demonstrated the stronger aggregation by $\pi-\pi$ stacking of Azo units in the polymer side chain. 

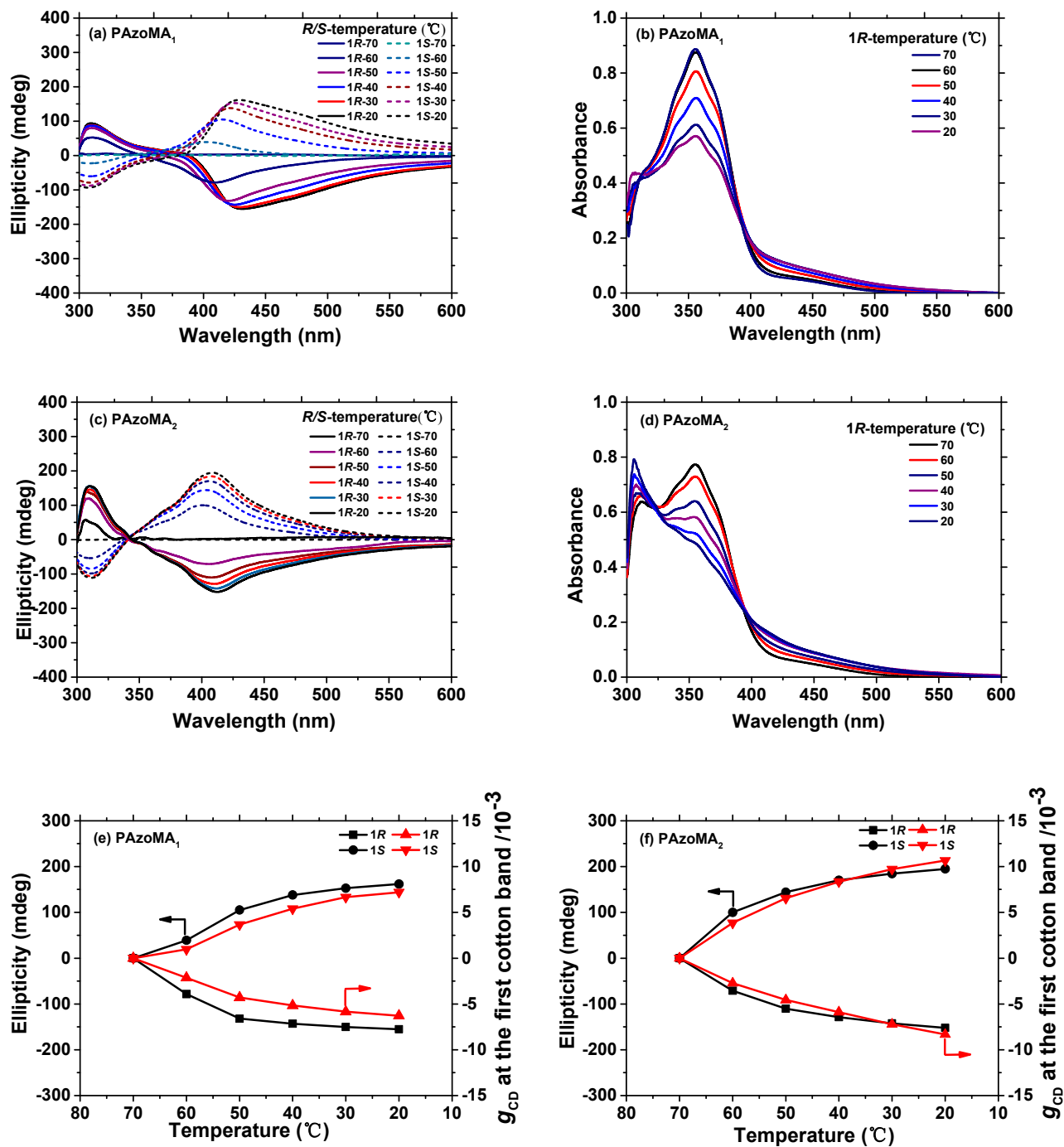

Figure 1. The circular dichroism $(\mathrm{CD})(\mathbf{a}, \mathbf{c}), \mathrm{UV}$-vis spectra $(\mathbf{b}, \mathbf{d})$ and maximum $\mathrm{CD}$ and $g_{\mathrm{CD}}$ values $(\mathbf{e}, \mathbf{f})$ of the side-chain Azo-containing polymer aggregates with the temperature decreasing from $70^{\circ} \mathrm{C}$ to $20^{\circ} \mathrm{C}$. $(\mathbf{a}, \mathbf{b}, \mathbf{e})$ stand for PAzoMA $1,(\mathbf{c}, \mathbf{d}, \mathbf{f})$ stand for $\mathrm{PAzoMA}_{2}$. The concentration of polymer repeating units is $8.42 \times 10^{-5} \mathrm{~mol} \mathrm{~L}^{-1}$.

The above results can also be proved by dynamic light scattering (DLS). With the temperature decreasing from $70{ }^{\circ} \mathrm{C}$ to $20^{\circ} \mathrm{C}$, the size of the polymer aggregates becomes bigger, ranging from $0 \mathrm{~nm}$ to $810 \mathrm{~nm}$ in $R$-limonene, and from $0 \mathrm{~nm}$ to $784 \mathrm{~nm}$ in $S$-limonene (Figure 2). 


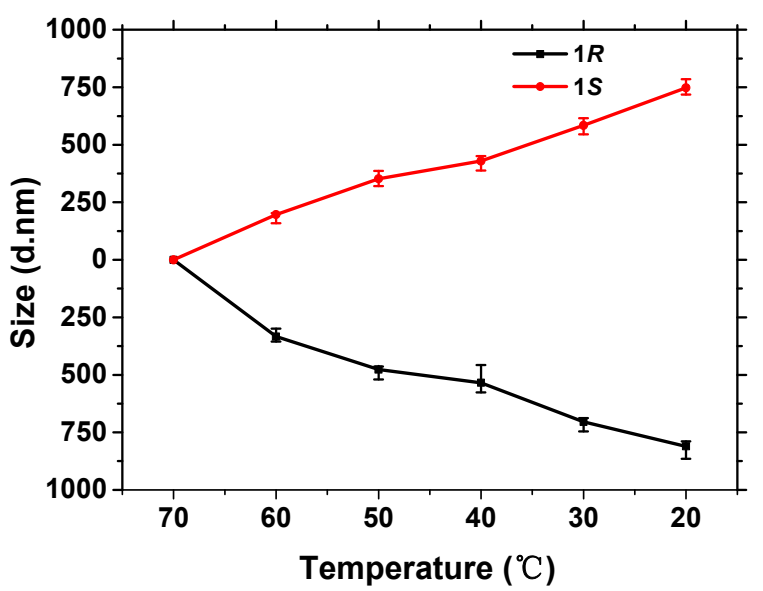

Figure 2. The dependence of the PAzoMA 1 aggregates' size on the temperature, which is decreasing from $70{ }^{\circ} \mathrm{C}$ to $20^{\circ} \mathrm{C}$. These date are taken from Figure S4a,b. The concentration of polymer repeating units is the same as in Figure 1.

\subsection{Supramolecular Chirality of Polymer Solid Films}

The liquid-crystalline phase transition of side-chain Azo-containing polymers has been intensively investigated in many groups [37-39]. According to the DSC results (Figure S5), the glass transition temperature is around $65^{\circ} \mathrm{C}$ for both PAzoMA 1 and PAzoMA $\mathrm{P}_{2}$. In the case of a polymer solid film, a small amount of $1 R$ or $1 S$ limonene $(0.1 \mathrm{~mL})$ was added to the quartz cell. After that, the polymer solid film was fixed in the cell and suspended above the limonene surface. When $1 R$ was added, the intense negative CD signal related to the $\pi-\pi^{*}$ electronic transition of the trans-Azo chromophore was observed at $60{ }^{\circ} \mathrm{C}$, indicating that the optically active polymer film was successfully produced by limonene vapor. The absorption of the $\pi-\pi^{*}$ band began to decrease in the UV-vis spectra (Figure S6) when the temperature reached $70{ }^{\circ} \mathrm{C}$ beyond $T_{\mathrm{g}}$. The chiral signal in the $\mathrm{CD}$ spectra disappeared immediately at the same time. The reason may be that the chain segment of the polymer would begin to move randomly after heating above its $T_{\mathrm{g}}$ temperature, which would destroy the well-organized helical $\pi-\pi$ stacking of the Azo units induced by limonene vapor. Similar results were found in the thin films of achiral liquid crystal polymers induced by CPL [35]. Interestingly, when the polymer films were cooled to $60^{\circ} \mathrm{C}$ again, the polymer chains cooled down in the environment of the chiral vapors, and the chiral aggregates could re-form again. Chiral signals similar to the previous intensity were observed in the CD spectrum. We successfully built a chiral switch of the polymer solid film by alternately changing the temperature between $70{ }^{\circ} \mathrm{C}$ and $60^{\circ} \mathrm{C}$. Five switching cycles were tested, and the obvious decline of the maximum absolute $\mathrm{CD}$ amplitude was not found, as presented in Figure 3. Almost intense mirror-image $C D$ spectra were obtained when $1 R$ was replaced by $1 S$.
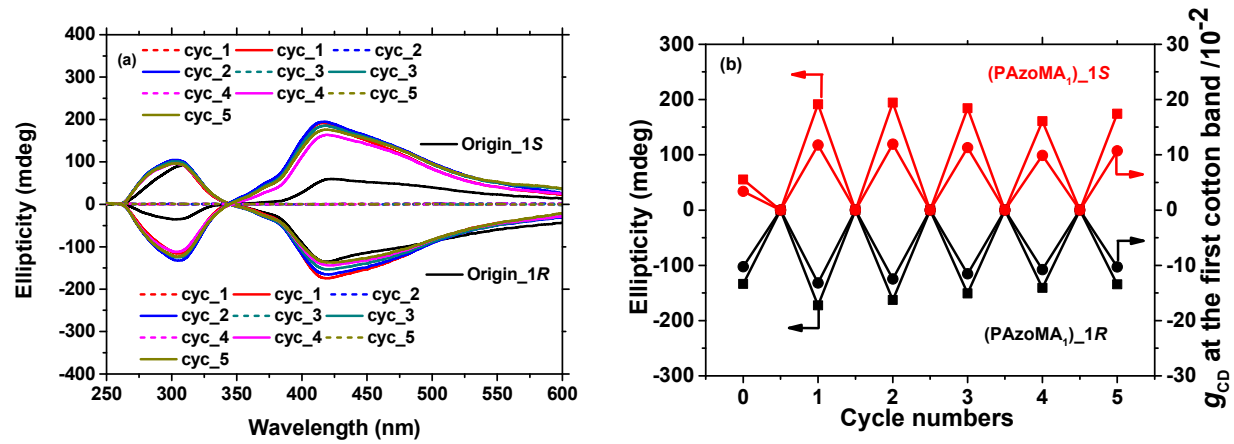

Figure 3. Changes in $\mathrm{CD}$ spectra (a) and the maximum $\mathrm{CD}$ and $g_{\mathrm{CD}}$ values (b) of PAzoMA $\mathrm{A}_{1}$ films during heating to $70{ }^{\circ} \mathrm{C}$ and cooling to $60{ }^{\circ} \mathrm{C}$ five times. 


\subsection{Chiral Memory Property of Azo-Containing Polymer Film}

Due to the strong molecular interaction occurring in the polymer chains in the solid state, the polymer films always present a superior chiral memory performance [40]. In this case, the supramolecular chirality was firstly induced in thin films of the achiral side chain Azo-containing polymers by limonene vapors. After that, the residual limonene molecular was removed at $40{ }^{\circ} \mathrm{C}$ under vacuum for $24 \mathrm{~h}$, and then stored in a fume hood for several months. The content of limonene was measured by ${ }^{1} \mathrm{H}$ NMR and described in Figure S7. There would be a slight attenuation of the CD signals in the PAzoMA films at the beginning, and then, the signals could maintain a stable intensity for several months. The results can be proved in Figure 4.
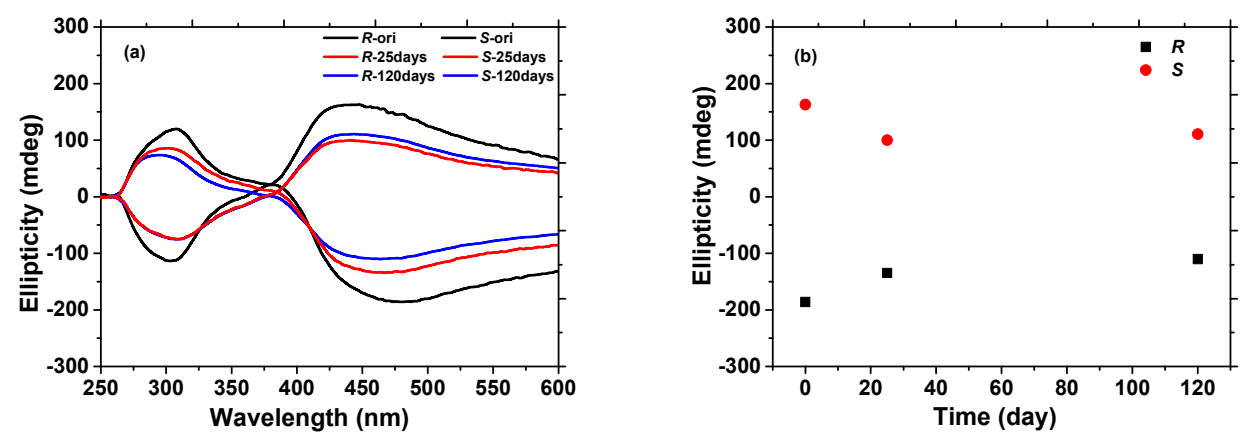

Figure 4. Changes in the CD spectra (a) and the maximum $\mathrm{CD}(\mathbf{b})$ of the $\mathrm{PAzoMA}_{1}$ aggregates in the films without the existence of limonene.

\subsection{Chiral Amplification}

By varying the enantiomeric excess (ee) of chiral limonene, the possibility of chiral amplification in the aggregation of Azo units were investigated. While keeping the temperature of the system as a constant $\left(60^{\circ} \mathrm{C}\right)$, the linear plot of maximum CD and $g_{\mathrm{CD}}$ values (Figure 5) demonstrated that the chiral aggregation of Azo units was linearly controlled by the respective molar ratio of enantiomers $(1 R / 1 S)$. No obvious chiral amplification occurred while changing the enantiomeric excess (ee) of the chiral solvent. A similar result was also found in the polymer solid films. The chiral signals of the aggregates in the thin films can be adjusted by changing the ee of the chiral limonene vapors. Similar results were also reported in the limonene-induced supramolecular chirality of $\pi$-conjugated main-chain polymers [9,40] and side-chain polymers [27,29].
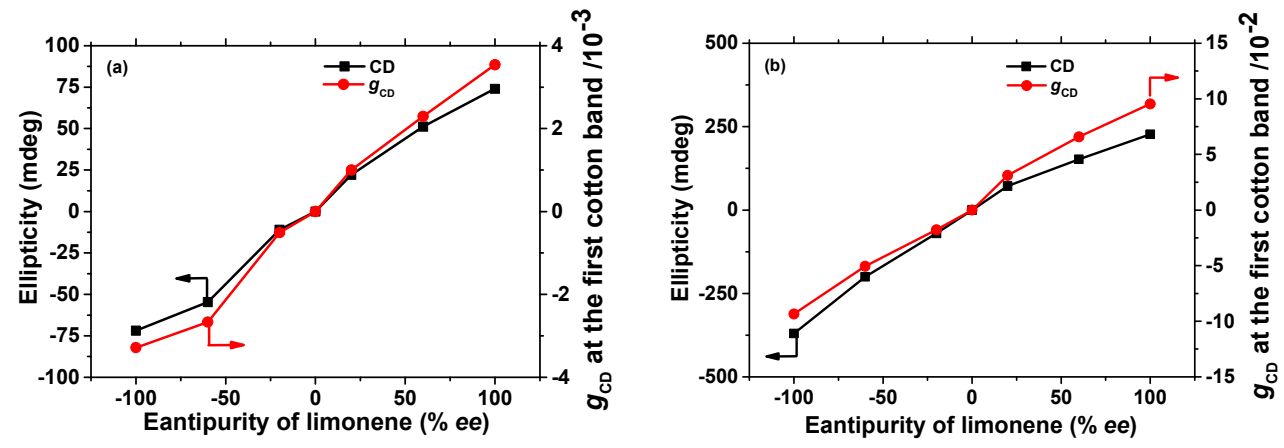

Figure 5. Plots of maximum $\mathrm{CD}$ and $g_{\mathrm{CD}}$ values of the PAzoMA 1 in the solution (a) and films (b) with different enantiopurity of limonene. These data were taken from Figure S8. The solution and films were both heated to $70^{\circ} \mathrm{C}$ and measured after being cooled down to $60^{\circ} \mathrm{C}$. 


\section{Conclusions}

In summary, the supramolecular chirality was successfully introduced to achiral side chain Azo-containing polymers by heating-cooling treatment in pure limonene solution. With the decrease of temperature, the chiral signals of aggregates tended to increase. Besides, the supramolecular chirality of the polymer solid films could be induced by limonene vapors. The chiral supramolecular structure can be destroyed by heating the film above the glass transition temperature $\left(T_{\mathrm{g}}\right)$ of the polymer due to the irregular movements of the polymer chains. Meanwhile, it can be recovered by cooling down the film in the environment of limonene vapors. This reversible chiral—achiral switching process can be repeated more than five times. Furthermore, the supramolecular chirality can be perfectly memorized in the solid state. We successfully achieved the supramolecular chirality of the achiral side chain Azo-containing polymers in the neat chiral limonene and the thin films induced by chiral limonene vapor.

Supplementary Materials: The following are available online at http:/ / www.mdpi.com/2073-4360/10/6/612/s1. Figure S1: ${ }^{1} \mathrm{H}$ NMR (a) and ${ }^{13} \mathrm{C}$ NMR (b) spectra of AzoMA, Figure S2: GPC curves of PAzoMAs, Figure S3: UV-vis spectra of Azo-containing polymer aggregates in $1 \mathrm{~S}$ with the temperature decreasing from $70{ }^{\circ} \mathrm{C}$ to $20^{\circ} \mathrm{C}$. (a) stands for PAzoMA 1 and (b) stands for PAzoMA 2 , Figure S4: The dependence of PAzoMA 1 aggregates size in limonene under different temperature, Figure S5: DSC heating curves of PAzoMA ${ }_{1}$ and PAzoMA 2 , Figure S6: UV-vis spectra of the polymer films under limonene vapors during the process of changing the temperature, Figure S7: ${ }^{1} \mathrm{H}$ NMR spectra of the residual limonene on the polymer films after chiral induction (a) and placed in the fume hood for 45 days (b), Figure S8: Changes in CD and UV-vis spectra of Azo polymer (PAzoMA 1 ) aggregates in the solution (a) and films (b) with different enantiopurity of limonene.

Author Contributions: T.M., L.Y., X.C. and Y.Z. performed the experiments. T.M. and L.Y. analyzed the date and wrote the manuscript. W.H., W.Z. and X.Z. designed the experimental plan and revised the manuscript. T.M. and L.Y. contributed equally to this work.

Acknowledgments: The authors are grateful for the financial support from the National Nature Science Foundation of China (21574089), the Priority Academic Program Development (PAPD) of Jiangsu Higher Education Institutions and the Program of Innovative Research Team of Soochow University.

Conflicts of Interest: The authors declare no conflict of interest.

\section{References}

1. Nafie, L.A. Physical chemistry handedness detected by microwaves. Nature 2013, 497, 446-448. [CrossRef] [PubMed]

2. Cornelissen, J.J.L.M.; Rowan, A.E.; Nolte, R.J.M.; Sommerdijk, N.A.J.M. Chiral architectures from macromolecular building blocks. Chem. Rev. 2001, 101, 4039-4070. [CrossRef] [PubMed]

3. Okoshi, K.; Sakurai, S.; Ohsawa, S.; Kumaki, J.; Yashima, E. Control of main-chain stiffness of a helical poly(phenylacetylene) by switching on and off the intramolecular hydrogen bonding through macromolecular helicity inversion. Angew. Chem. Int. Ed. 2006, 45, 8173-8176. [CrossRef] [PubMed]

4. Bai, S.; Debnath, S.; Javid, N.; Frederix, P.W.; Fleming, S.; Pappas, C.; Ulijn, R.V. Differential self-assembly and tunable emission of aromatic peptide bola-amphiphiles containing perylene bisimide in polar solvents including water. Langmuir 2014, 30, 7576-7584. [CrossRef] [PubMed]

5. Lee, D.; Jin, Y.J.; Kim, H.; Suzuki, N.; Fujiki, M.; Sakaguchi, T.; Kim, S.K.; Lee, W.E.; Kwak, G. Solvent-to-polymer chirality transfer in intramolecular stack structure. Macromolecules 2012, 45, 5379-5386. [CrossRef]

6. Marinelli, F.; Sorrenti, A.; Corvaglia, V.; Leone, V.; Mancini, G. Molecular description of the propagation of chirality from molecules to complex systems: Different mechanisms controlled by hydrophobic interactions. Chem. Eur. J. 2012, 18, 14680-14688. [CrossRef] [PubMed]

7. Sobczuk, A.A.; Tsuchiya, Y.; Shiraki, T.; Tamaru, S.; Shinkai, S. Creation of chiral thixotropic gels through a crown-ammonium interaction and their application to a memory-erasing recycle system. Chem. Eur. J. 2012, 18, 2832-2838. [CrossRef] [PubMed]

8. Akagi, K. Helical polyacetylene: Asymmetric polymerization in a chiral liquid-crystal field. Chem. Rev. 2009, 109, 5354-5401. [CrossRef] [PubMed] 
9. Zhang, W.; Yoshida, K.; Fujiki, M.; Zhu, X.L. Unpolarized-light-driven amplified chiroptical modulation between chiral aggregation and achiral disaggregation of an azobenzene-alt-fluorene copolymer in limonene. Macromolecules 2011, 44, 5105-5111. [CrossRef]

10. Hase, Y.; Nagai, K.; Iida, H.; Maeda, K.; Ochi, N.; Sawabe, K.; Sakajiri, K.; Okoshi, K.; Yashima, E. Mechanism of helix induction in poly(4-carboxyphenyl isocyanide) with chiral amines and memory of the macromolecular helicity and its helical structures. J. Am. Chem. Soc. 2009, 131, 10719-10732. [CrossRef] [PubMed]

11. Nakano, Y.; Ichiyanagi, F.; Naito, M.; Yang, Y.G.; Fujiki, M. Chiroptical generation and inversion during the mirror-symmetry-breaking aggregation of dialkylpolysilanes due to limonene chirality. Chem. Commun. 2012, 48, 6636-6638. [CrossRef] [PubMed]

12. Wang, L.; Yin, L.; Zhang, W.; Zhu, X.; Fujiki, M. Circularly polarized light with sense and wavelengths to regulate azobenzene supramolecular chirality in optofluidic medium. J. Am. Chem. Soc. 2017, 139, 13218-13226. [CrossRef] [PubMed]

13. Wang, Y.; Sakamoto, T.; Nakano, T. Molecular chirality induction to an achiral pi-conjugated polymer by circularly polarized light. Chem. Commun. 2012, 48, 1871-1873. [CrossRef] [PubMed]

14. Zou, G.; Jiang, H.; Kohn, H.; Manaka, T.; Iwamoto, M. Control and modulation of chirality for azobenzene-substituted polydiacetylene $\mathrm{lb}$ films with circularly polarized light. Chem. Commun. 2009, 5627-5629. [CrossRef] [PubMed]

15. Yuan, J.; Liu, M.H. Chiral molecular assemblies from a novel achiral amphiphilic 2-(heptadecyl) naphtha[2,3]imidazole through interfacial coordination. J. Am. Chem. Soc. 2003, 125, 5051-5056. [CrossRef] [PubMed]

16. Shen, Z.C.; Wang, T.Y.; Shi, L.; Tang, Z.Y.; Liu, M.H. Strong circularly polarized luminescence from the supramolecular gels of an achiral gelator: Tunable intensity and handedness. Chem. Sci. 2015, 6, 4267-4272. [CrossRef] [PubMed]

17. Bosnich, B. Asymmetric syntheses asymmetric transformations and asymmetric inductions in an optically active solvent. J. Am. Chem. Soc. 1967, 89, 6143-6148. [CrossRef]

18. Jonkheijm, P.; Miura, A.; Zdanowska, M.; Hoeben, F.J.; De Feyter, S.; Schenning, A.P.; De Schryver, F.C.; Meijer, E.W. Pi-conjugated oligo-( $p$-phenylenevinylene) rosettes and their tubular self-assembly. Angew. Chem. Int. Ed. 2004, 43, 74-78. [CrossRef] [PubMed]

19. Makiguchi, W.; Kobayashi, S.; Furusho, Y.; Yashima, E. Formation of a homo double helix of a conjugated polymer with carboxy groups and amplification of the macromolecular helicity by chiral amines sandwiched between the strands. Angew. Chem. Int. Ed. 2013, 52, 5275-5279. [CrossRef] [PubMed]

20. Thomas, R.; Tamaoki, N. Chirality transfer from chiral solvents and its memory in an azobenzene derivative exhibiting photo-switchable racemization. Org. Biomol. Chem. 2011, 9, 5389-5393. [CrossRef] [PubMed]

21. Green, M.M.; Khatri, C.; Peterson, N.C. A macromolecular conformational change driven by a minute chiral solvation energy. J. Am. Chem. Soc. 1993, 115, 4941-4942. [CrossRef]

22. Nakashima, H.; Koe, J.R.; Torimitsu, K.; Fujiki, M. Transfer and amplification of chiral molecular information to polysilylene aggregates. J. Am. Chem. Soc. 2001, 123, 4847-4848. [CrossRef] [PubMed]

23. Nakano, Y.; Liu, Y.; Fujiki, M. Ambidextrous circular dichroism and circularly polarised luminescence from poly(9,9-di-n-decylfluorene) by terpene chirality transfer. Polym. Chem. 2010, 1, 460-469. [CrossRef]

24. Kawagoe, Y.; Fujiki, M.; Nakano, Y. Limonene magic: Noncovalent molecular chirality transfer leading to ambidextrous circularly polarised luminescent pi-conjugated polymers. New J. Chem. 2010, 34, 637-647. [CrossRef]

25. Fujiki, M.; Kawagoe, Y.; Nakano, Y.; Nakao, A. Mirror-symmetry-breaking in poly[(9,9-di- $n$-octylfluorenyl2,7-diyl)-alt-biphenyl] (pf8p2) is susceptible to terpene chirality, achiral solvents, and mechanical stirring. Molecules 2013, 18, 7035-7057. [CrossRef] [PubMed]

26. Yashima, E.; Matsushima, T.; Okamoto, Y. Chirality assignment of amines and amino alcohols based on circular dichroism induced by helix formation of a stereoregular poly((4-carboxyphenyl)acetylene) through acid-base complexation. J. Am. Chem. Soc. 1997, 119, 6345-6359. [CrossRef]

27. Jiang, S.Q.; Zhao, Y.; Wang, L.; Yin, L.B.; Zhang, Z.B.; Zhu, J.; Zhang, W.; Zhu, X.L. Photocontrollable induction of supramolecular chirality in achiral side chain azo-containing polymers through preferential chiral solvation. Polym. Chem. 2015, 6, 4230-4239. [CrossRef] 
28. Lustig, S.R.; Everlof, G.J.; Jaycox, G.D. Stimuli-responsive polymers. 5. Azobenzene modified polyaramides containing atropisomeric binaphthyl linkages: Tuning chiroptical behavior with light and heat. Macromolecules 2001, 34, 2364-2372. [CrossRef]

29. Yin, L.; Zhao, Y.; Liu, M.; Zhou, N.C.; Zhang, W.; Zhu, X.L. Induction of supramolecular chirality by chiral solvation in achiral Azo-containing polymers with different spacer lengths and push-pull electronic substituents: Where will chiral induction appear? Polym. Chem. 2017, 8, 1906-1913. [CrossRef]

30. Yin, L.; Zhao, Y.; Jiang, S.Q.; Wang, L.B.; Zhang, Z.B.; Zhu, J.; Zhang, W.; Zhu, X.L. Preferential chiral solvation induced supramolecular chirality in optically inactive star Azo-containing polymers: Photocontrollability, chiral amplification and topological effects. Polym. Chem. 2015, 6, 7045-7052. [CrossRef]

31. Zhao, Y.; Chen, H.; Yin, L.; Cheng, X.; Zhang, W.; Zhu, X. Chirality induction of achiral polydialkylfluorenes by chiral solvation: Odd-even and side chain length dependence. Polym. Chem. 2018, 9, 2295-2301. [CrossRef]

32. Yashima, E.; Maeda, K. Chirality-responsive helical polymers. Macromolecules 2008, 41, 3-12. [CrossRef]

33. Rizzo, P.; Montefusco, T.; Guerra, G. Chiral optical films based on achiral chromophore guests. J. Am. Chem. Soc. 2011, 133, 9872-9877. [CrossRef] [PubMed]

34. Kim, M.J.; Shin, B.G.; Kim, J.J.; Kim, D.Y. Photoinduced supramolecular chirality in amorphous azobenzene polymer films. J. Am. Chem. Soc. 2002, 124, 3504-3505. [CrossRef] [PubMed]

35. Wu, Y.L.; Natansohn, A.; Rochon, P. Photoinduced chirality in thin films of achiral polymer liquid crystals containing azobenzene chromophores. Macromolecules 2004, 37, 6801-6805. [CrossRef]

36. Zhang, Y.; Zhang, W.; Chen, X.; Cheng, Z.; Wu, J.; Zhu, J.; Zhu, X. Synthesis of novel three-arm star azo side-chain liquid crystalline polymer via atrp and photoinduced surface relief gratings. J. Polym. Sci. Part A Polym. Chem. 2008, 46, 777-789. [CrossRef]

37. Han, D.H.; Tong, X.; Zhao, Y.; Galstian, T.; Zhao, Y. Cyclic azobenzene-containing side-chain liquid crystalline polymers: Synthesis and topological effect on mesophase transition, order, and photoinduced birefringence. Macromolecules 2010, 43, 3664-3671. [CrossRef]

38. Zhu, Y.; Wang, X.G. Synthesis and photoresponsive properties of two liquid crystalline polymers bearing branched azobenzene-containing side chains. Polym. Chem. 2013, 4, 5108-5118. [CrossRef]

39. Wang, T.J.; Li, X.; Dong, Z.J.; Huang, S.; Yu, H.F. Vertical orientation of nanocylinders in liquid-crystalline block copolymers directed by light. ACS Appl. Mater. Interfaces 2017, 9, 24864-24872. [CrossRef] [PubMed]

40. Zhao, Y.; Rahirn, N.A.A.; Xia, Y.J.; Fujiki, M.; Song, B.; Zhang, Z.B.; Zhang, W.; Zhu, X.L. Supramolecular chirality in achiral polyfluorene: Chiral gelation, memory of chirality, and chiral sensing property. Macromolecules 2016, 49, 3214-3221. [CrossRef] 